\title{
Optimal System for Warm Standby Components in the Presence of Standby Switching Failures, Two Types of Failures and General Repair Time
}

\author{
Mohamed Salah EL-Sherbeny \\ Department of Mathematics, Faculty of Science, Helwan University P. O. Box 11795, Cairo, Egypt
}

\begin{abstract}
Two different system configurations with warm standby components, standby switching failures, two types of failures "common cause failure and hardware failure" and general repair are compared based on the availability. The time-tofailure for each of the primary and warm standby components are assumed to follow the exponential distribution. Laplace transforms of state probability equations are developed by using the supplementary variable technique. We develop the explicit expressions for the steady-state availability, $A v$, for two configurations. For all configurations, comparisons are made for specific values of distribution parameters and of the cost of the components. The configurations are ranked based on $A v$ and cost/benefit, for three various repair time distributions: Gamma (G), Weibull (W) and Lognormal (L), where benefit is $A v$.
\end{abstract}

\section{Keywords}

Availability; Standby switching failures; Common cause failure "CCF"; Supplementary variable; General repair times.

\section{INTRODUCTION}

In this paper we use a supplementary variable technique to study the availability analysis of two different series system configurations with warm standby units, imperfect switch and two types of failures. The steady-state availability Av has widely been analyzed in the literature because of its prevalence in power plants, manufacturing systems, and industrial systems. Maintaining a high or required level of availability is often an essential requisite. El-Said and ElSherbeny [2] studied two systems, each system with two parallel components. The second system differs from the first system due to the additional feature of preventive maintenance. The two systems are analyzed under the assumption that the failure, replacement and preventive maintenance times of the units are assumed to be arbitrarily distributed. Wang and Kuo [7] dealt the reliability, the availability, and the cost/benefit analysis of four different series system configurations with mixed standby (include cold standby and warm standby) components. The stochastic analysis of a non-identical two-unit parallel system with common-cause failure by graphical evaluation and review technique (GERT) considered by Sridharan and Kalyani [5]. Gaikowsky, et al. [4] and Wang and Pearn [8] analyzed the series systems with cold standby components and warm standby components, respectively, where the repair time distribution of the server is assumed to be exponentially distributed. Shen, et al. [6] discussed exponential asymptotic property of the solution of a parallel repairable system with warm standby under common cause failure. Dhillon and Anude [1] studied Common-cause failure analysis of a nonidentical unit parallel system with arbitrarily distributed repair times. A standby component is called a "warm standby" if its failure rate is nonzero and is less than the failure rate of a primary component. Primary and warm standby components can be considered to be repairable. A CCF is defined as the failure of single unit or multiple units due to a single common-cause. Some of the CCF may occur due to the following reasons.

- abnormal environmental conditions, e.g. temperature, pressure;

- defective design; and

- natural catastrophe like fire, ... etc.

The problem considered in this paper is more different than the works of M. Salah EL-Sherbeny [3]. We first present a recursive method, using the supplementary variable technique and treating the supplementary variable as the elapsed repair time, to develop the $A v_{i}$, for configuration i, where i $=1,2$. Next, for each configuration, the explicit expressions for the Av for three different repair time distributions such as Gamma (G), Weibull (W) and Lognormal (L) are provided. Finally, we rank two configurations for the Av based on assumed numerical values given to the system parameters.

\section{ASSUMPTIONS AND MODEL DESCRIPTION}

We consider a power plant of $30 \mathrm{MW}$ satisfying the following assumptions:

1. The system comprises of operative components and warm standby components.

2. The generators are available in both 30 and $15 \mathrm{MW}$.

3. Standby generators are always necessary in case of failure.

4. In case of failure of an operative component, it is immediately replaced by a standby component if it is available.

5. The switch from standby component to operative component is imperfect switch, with probability $\theta$.

6 . The system suffers two types of failures, namely, hardware and common-cause failure.

7. The failures are statistically independent.

8. Failed system repair times are assumed to be arbitrarily distributed.

9. The common-cause and other failure rates are constant.

10. A unit's repair rate is constant.

11. A repaired unit (or system) is assumed to be as good as new.

The above assumptions are common to all of the following two configurations.

be justified, not ragged. 


\subsection{Configuration descriptions}

The first configuration composes of one operative $30 \mathrm{MW}$ component and one standby $30 \mathrm{MW}$ component. The second configuration contains two primary $15 \mathrm{MW}$ components and one standby $15 \mathrm{MW}$ component.

Notation

The following symbols are used in this paper:

$\lambda_{C_{i}}$ Constant common-cause failure rate from state $i$ to state 3 ; for $i=0,1$.

$\lambda$ Constant hardware failure rate of operative unit.

$\alpha$ Constant failure rate of the warm standby.

$\mu \quad$ Constant repair rate of a failed unit in state 1 .

$P_{i} \quad$ The steady state probability that the system is in state $i ; i=0,1,23$.

$P_{i}(x)$ The probability that the system is in state $\mathrm{i}$ at time $\mathrm{t}$; for $\mathrm{i}=0,1,2,3$.

$(1-\theta) \quad \mathrm{P}$ [switching device is found to be good when needed].

$E_{k}(x)$ The mean time to system repair that the failed system is in state $k$ and has an elapsed repair time of $x$; for $k=2,3$.

$\mu_{k}(x)$ Time-dependent system repair rate when the system is in state $k$ and has an elapsed repair time of $x$; for $k=2,3$.

$P_{k}(x, t)$ The probability that the failed system is in state $k$ and has an elapsed repair time of $x$; for $k=2,3$.

$f_{k}(x)$ Probability density function ( $\left.p d f\right)$ of the system repair time when the system is in state $k$ and has an elapsed repair time of $x ; k=2,3$.

\subsection{Cost-benefit factor}

We assume that the size-proportional costs for the primary components and warm standby components are given in Table 1. With this, we calculate the costs for each configuration $i(i=1,2)$ shown in Table 2. Let $C_{i}$ be the cost of the configuration $i$, and $B_{i}$ be the benefit of the configuration $i$, where $B_{i}$ is the $A v_{i}$.

Table 1.The cost for the primary and warm standby components

\begin{tabular}{|r|r|}
\hline Component & Cost(in \$) \\
\hline Primary 30 MW & $30 \times 10^{6}$ \\
\hline Primary 15 MW & $15 \times 10^{6}$ \\
\hline Warm standby 30 MW & $10 \times 10^{6}$ \\
\hline Warm standby 15 MW & $5 \times 10^{6}$ \\
\hline
\end{tabular}

Table 2. The costs for each Model $i, i=1,2$.

\begin{tabular}{|l|l|}
\hline Models & Cost(in \$) \\
\hline Model 1 & $40 \times 10^{6}$ \\
\hline Model 2 & $35 \times 10^{6}$ \\
\hline
\end{tabular}

\section{Availability analysis of the system}

\subsection{Availability for configuration 1}

Descriptive Equations for configuration 1:

$$
\begin{aligned}
& {\left[\frac{d}{d t}+\lambda+\alpha+\lambda_{C_{0}}\right] P_{0}(t)=\mu P_{1}(t)+\sum_{k=2}^{3} \int_{0}^{\infty} \mu_{k}(x) P_{k}(x, t) d x,} \\
& {\left[\frac{d}{d t}+\lambda+\mu+\lambda_{C_{1}}\right] P_{1}(t)=((1-\theta) \lambda+\alpha) P_{0}(t),} \\
& {\left[\frac{\partial}{\partial t}+\frac{\partial}{\partial x}+\mu_{3}(x)\right] P_{3}(x, t)=0,} \\
& {\left[\frac{\partial}{\partial t}+\frac{\partial}{\partial x}+\mu_{2}(x)\right] P_{2}(x, t)=0 .}
\end{aligned}
$$

The associated boundary conditions are as follows:

$$
\begin{aligned}
& P_{2}(0, t)=\lambda \theta P_{0}(t)+\lambda P_{1}(t), \\
& P_{3}(0, t)=\lambda_{C_{0}} P_{0}(t)+\lambda_{C_{1}} P_{1}(t) .
\end{aligned}
$$

When $t=0$ then $P_{0}(0)=1$ and $P_{1}(0)=P_{k}(x, 0)=0$; for $k=2,3$.

Steady-state availability analysis as $t \rightarrow \infty$, the equations (1-4) reduce to equation (7-9), respectively.

$$
\begin{aligned}
& \left(\lambda+\alpha+\lambda_{C_{0}}\right) P_{0}-\mu P_{1}=\sum_{k=2}^{3} \int_{0}^{\infty} \mu_{k}(x) P_{k}(x, t) d x, \\
& \left(\lambda+\mu+\lambda_{C_{1}}\right) P_{1}-((1-\theta) \lambda+\alpha) P_{0}=0, \\
& \frac{\partial P_{k}(x)}{\partial x}=-\mu_{k}(x) P_{k}(x) . \forall k=2,3 .
\end{aligned}
$$

Similarly, the boundary conditions become:

$P_{2}(0)=\lambda \theta P_{0}+\lambda P_{1}$,

$P_{3}(0)=\lambda_{C_{0}} P_{0}+\lambda_{C_{1}} P_{1}$.

$P_{i}$ is the steady-state probability that the system is in state $i$, for $i=0, \ldots, 3$.

and $P_{k}=\int_{0}^{\infty} P_{k}(x) d x$, for $k=2,3$.

Also

$\sum_{i=0}^{3} P_{i}=1$.

Solving differential equation (9), we get

$$
P_{k}(x)=P_{k}(0) \exp \left(-\int_{0}^{x} \mu_{k}(w) d w\right) \text {.for } k=2,3 \text {. }
$$

Thus, from equation (12) and (14), we have

$$
\begin{aligned}
& P_{2}=\int_{0}^{\infty} P_{2}(x) d x \\
& P_{2}=\int_{0}^{\infty} P_{2}(0) \exp \left(-\int_{0}^{x} \mu_{2}(w) d w\right) d x \\
& P_{2}=\left(\lambda \theta P_{0}+\lambda P_{1}\right) E_{2}(x)
\end{aligned}
$$

Similarly,

$$
\begin{aligned}
& P_{3}=\int_{0}^{\infty} P_{3}(x) d x \\
& P_{3}=\int_{0}^{\infty} P_{3}(0) \exp \left(-\int_{0}^{x} \mu_{3}(w) d w\right) d x \\
& P_{3}=\left(\lambda_{C_{0}} P_{0}+\lambda_{C_{1}} P_{1}\right) E_{3}(x)
\end{aligned}
$$

where

$E_{k}(x)=\int_{0}^{\infty} \exp \left(-\int_{0}^{x} \mu_{k}(w) d w\right) d x$

$E_{2}(x)$ and $E_{3}(x)$ are the mean times to repair from state 2 to state 0 , from state 3 to state 0 , respectively.

Solving the set equations (8), (13), (15) and (16), we get the following steady state probabilities: 
$P_{0}=\frac{1}{a_{0}+a_{1} r}$,

$P_{1}=\frac{r}{a_{0}+a_{1} r}$,

$P_{2}=\frac{\lambda(\theta+r) E_{2}(x)}{a_{0}+a_{1} r}$,

$P_{3}=\frac{\left(\lambda_{C_{0}}+\lambda_{C_{1}} r\right) E_{3}(x)}{a_{0}+a_{1} r}$.

where

$a_{0}=\left(1+\lambda \theta E_{2}(x)+\lambda_{C_{0}} E_{3}(x)\right), \quad a_{1}=\left(1+\lambda E_{2}(x)+\lambda_{C_{1}} E_{3}(x)\right)$

and $r=\frac{(1-\theta) \lambda+\alpha}{\lambda+\mu+\lambda_{C_{1}}}$

The system steady state availability is

$A v_{1}=P_{0}+P_{1}=\frac{1+r}{a_{0}+a_{1} r}$

Similarly, the system steady state unavailability is given by

$U A v_{1}=P_{2}+P_{3}=\frac{\lambda(\theta+r) E_{2}(x)+\left(\lambda_{C_{0}}+r \lambda_{C_{1}}\right) E_{3}(x)}{a_{0}+a_{1} r}$.

\subsubsection{Special cases}

\section{Case I}

If the system repair time $x$ is Gamma distributed and probability density function (pdf) of the repair time is given by

$$
\begin{aligned}
& f_{k}(x)=\frac{\mu_{k}^{\beta} x^{\beta-1}}{\Gamma(\beta)} \exp \left(-\mu_{k} x\right) . \\
& \quad \text { for } k=2,3 ; x \geq 0, \beta>0, \mu_{j}>0 .
\end{aligned}
$$

where $\beta$ and $\mu_{j}$ are two parameters of Gamma distribution.

Thus, the mean time to repair $E_{k}(x)$ is

$$
E_{k}(x)=\frac{\beta}{\mu_{k}} . k=2,3 \text {. }
$$

Substituting Equation (24) into equations (21), we get the following resulting system steady state availability for the Gamma repair time distribution:

$$
A v_{1}(G)=\frac{1+r}{a_{4}+a_{5} r} \text {. }
$$

where

$$
a_{4}=\left(1+\frac{\lambda \theta \beta}{\mu_{2}}+\frac{\lambda_{C_{0}} \beta}{\mu_{3}}\right) \text {, and } a_{5}=\left(1+\frac{\lambda \beta}{\mu_{2}}+\frac{\lambda_{C_{1}} \beta}{\mu_{3}}\right) \text {. }
$$

\section{Case II}

If the system repair time $x$ is Weibull distributed and probability density function (pdf) of the repair time is given by

$$
\begin{aligned}
& f_{k}(x)=\mu_{k}^{\beta} \beta x^{\beta-1} \exp \left(-\mu_{k}^{\beta} x^{\beta}\right) . \\
& \quad \text { for } k=2,3 ; x \geq 0, \beta>0, \mu_{j}>0 .
\end{aligned}
$$

where $\beta$ and $\mu_{j}$ are two parameters of Weibull distribution.

Thus, the mean time to repair $E_{k}(x)$ is
$E_{k}(x)=\frac{\phi}{\mu_{k}} . k=2,3$.

where

$\phi=\Gamma(1+1 / \beta)$.

Substituting Equation (27) into equation (21), we get the following resulting system steady state availability for the Weibull repair time distribution:

$$
A v_{1}(W)=\frac{1+r}{a_{8}+a_{9} r} .
$$

where

$$
a_{8}=\left(1+\frac{\lambda \theta \phi}{\mu_{2}}+\frac{\lambda_{C_{0}} \phi}{\mu_{3}}\right) \text {, and } a_{9}=\left(1+\frac{\lambda \phi}{\mu_{2}}+\frac{\lambda_{C_{1}} \phi}{\mu_{3}}\right) \text {. }
$$

\section{Case III}

If the system repair time $x$ is lognormal distributed, the pdf of the repair time is defined by

$$
\begin{array}{r}
f_{k}(x)=\frac{1}{x \sigma_{k} \sqrt{2 \pi}} \exp \left(-\frac{\left(\ln x-\mu_{k}\right)^{2}}{2 \sigma_{k}^{2}}\right) . \\
\text { for } k=2,3 ; x \geq 0 .
\end{array}
$$

where $\mu_{k}$ and $\sigma_{k}$ are the distribution parameters (mean value and standard deviation of $\ln x$ respectively).

Thus, the mean time to repair $E_{k}(x)$ is

$E_{k}(x)=\phi_{k} \cdot k=2,3$.

where $\phi_{k}=\exp \left[\mu_{k}+\frac{\sigma_{k}^{2}}{2}\right]$

Substituting equation (30) into equation (21), we get the following resulting system steady state availability for the lognormal repair time distribution:

$A v_{1}(L)=\frac{1+r}{a_{12}+a_{13} r}$.

where

$$
a_{12}=\left(1+\lambda \theta \phi_{2}+\lambda_{C_{0}} \phi_{3}\right) \text {, and } a_{13}=\left(1+\lambda \phi_{2}+\lambda_{C_{1}} \phi_{3}\right)
$$

\subsection{Availability for configuration 2}

Descriptive Equations for configuration2: $\left[\frac{d}{d t}+2 \lambda+\alpha+\lambda_{C_{0}}\right] P_{0}(t)=\mu P_{1}(t)+\sum_{k=2}^{3} \int_{0}^{\infty} \mu_{k}(x) P_{k}(x, t) d x$,

$\left[\frac{d}{d t}+2 \lambda+\mu+\lambda_{C_{1}}\right] P_{1}(t)=(2 \lambda(1-\theta)+\alpha) P_{0}(t)$,
$\left[\frac{\partial}{\partial t}+\frac{\partial}{\partial x}+\mu_{k}(x)\right] P_{k}(x, t)=0$. for $k=2,3$.

The associated boundary conditions are as follows:

$$
\begin{aligned}
& P_{2}(0, t)=2 \lambda \theta P_{0}(t)+2 \lambda P_{1}(t), \\
& P_{3}(0, t)=\lambda_{C_{0}} P_{0}(t)+\lambda_{C_{1}} P_{1}(t) . \\
& \text { At } t=0 \text { then } P_{0}(0)=1 \text { and } P_{1}(0)=P_{k}(x, 0)=0 \text {; for } \\
& k=2,3 .
\end{aligned}
$$

Steady-state availability analysis

When $t \rightarrow \infty$, then the equations (32-34) reduce to equation (37-39), respectively. 
$\left(2 \lambda+\alpha+\lambda_{C_{0}}\right) P_{0}-\mu P_{1}=\sum_{k=2}^{3} \int_{0}^{\infty} \mu_{k}(x) P_{k}(x, t) d x$,

$\left(2 \lambda+\mu+\lambda_{C_{1}}\right) P_{1}-(2(1-\theta) \lambda+\alpha) P_{0}=0$,

$\frac{\partial P_{k}(x)}{\partial x}=-\mu_{k}(x) P_{k}(x) . \forall k=2,3$.

Similarly, the boundary conditions become:

$P_{2}(0)=2 \lambda \theta P_{0}+2 \lambda P_{1}$,

$P_{3}(0)=\lambda_{C_{0}} P_{0}+\lambda_{C_{1}} P_{1}$

$P_{i}$ is the steady-state probability that the system is in state $i$, for $i=0, \ldots, 3$.

and $P_{k}=\int_{0}^{\infty} P_{k}(x) d x$, for $k=2,3$.

Also

$\sum_{i=0}^{3} P_{i}=1$

We get the following steady state probabilities:

$P_{0}=\frac{1}{a_{2}+a_{3} r_{1}}$

$P_{1}=\frac{r_{1}}{a_{2}+a_{3} r_{1}}$

$P_{2}=\frac{2 \lambda\left(\theta+r_{1}\right) E_{2}(x)}{a_{2}+a_{3} r_{1}}$,

$P_{3}=\frac{\left(\lambda_{C_{0}}+\lambda_{C_{1}} r_{1}\right) E_{3}(x)}{a_{2}+a_{3} r_{1}}$.

where

$a_{2}=\left(1+2 \lambda \theta E_{2}(x)+\lambda_{C_{0}} E_{3}(x)\right)$,

$a_{3}=\left(1+2 \lambda E_{2}(x)+\lambda_{C_{1}} E_{3}(x)\right)$

and $r_{1}=\frac{2 \lambda(1-\theta)+\alpha}{2 \lambda+\mu+\lambda_{C_{1}}}$.

The system steady state availability is

$A v_{2}=P_{0}+P_{1}=\frac{1+r_{1}}{a_{2}+a_{3} r_{1}}$.

Similarly, the system steady state unavailability is given by

$$
U A v_{2}=P_{2}+P_{3}=\frac{2 \lambda\left(\theta+r_{1}\right) E_{2}(x)+\left(\lambda_{C_{0}}+r_{1} \lambda_{C_{1}}\right) E_{3}(x)}{a_{2}+a_{3} r_{1}}
$$

\subsubsection{Special cases}

For configuration 2, we also consider three special cases for different repair time distributions such as Gamma (G), Weibull (W), and lognormal (L). We provide the following explicit expressions for the $A v_{2}(G), A v_{2}(W)$ and $A v_{2}(L)$ for three different repair time distributions: Gamma, Weibull, and lognormal, respectively.

$A v_{2}(G)=\frac{1+r_{1}}{a_{6}+a_{7} r_{1}}$

where

$a_{6}=\left(1+\frac{2 \lambda \theta \beta}{\mu_{2}}+\frac{\lambda_{C_{0}} \beta}{\mu_{3}}\right)$, and $a_{7}=\left(1+\frac{2 \lambda \beta}{\mu_{2}}+\frac{\lambda_{C_{1}} \beta}{\mu_{3}}\right)$.
$A v_{2}(W)=\frac{1+r_{1}}{a_{10}+a_{11} r_{1}}$.

where

$a_{10}=\left(1+\frac{2 \lambda \theta \phi}{\mu_{2}}+\frac{\lambda_{C_{0}} \phi}{\mu_{3}}\right)$ and $a_{11}=\left(1+\frac{2 \lambda \phi}{\mu_{2}}+\frac{\lambda_{C_{1}} \phi}{\mu_{3}}\right)$.

$A v_{2}(L)=\frac{1+r_{1}}{a_{14}+a_{15} r_{1}}$

where

$a_{14}=\left(1+2 \lambda \theta \phi_{2}+\lambda_{C_{0}} \phi_{3}\right)$, and $a_{15}=\left(1+2 \lambda \phi_{2}+\lambda_{C_{1}} \phi_{3}\right)$.

4. Comparison between the two configurations

The purpose of this section is to compare $A v_{i}(i=1,2)$ for three different repair time distributions: Gamma, Weibull, and lognormal. Basically, we consider the following values:

$\lambda=0.2, \quad \lambda_{C_{1}}=0.3, \quad \lambda_{C_{0}}=0.35, \quad \mu=0.4, \quad \mu_{3}=0.6$, $\mu_{2}=0.5, \theta=0.7, \alpha=0.01, \beta=3.2$ and $\sigma_{2}=\sigma_{3}=2.5$.

4.1 Comparisons for the $A v_{i}$

Six cases are illustrated in Tables 3-8

Case 1. We fix $\lambda_{C_{1}}=0.3, \lambda_{C_{0}}=0.35, \mu=0.4, \mu_{3}=0.6$, $\mu_{2}=0.5, \theta=0.7, \alpha=0.01, \beta=3.2, \sigma_{2}=\sigma_{3}=2.5$ and vary the values of $\lambda$ from 0.2 to 1.6 .

Case 2. We fix $\lambda_{C_{1}}=0.3, \lambda=0.2, \mu=0.4, \mu_{3}=0.6$, $\mu_{2}=0.5, \quad \theta=0.7, \alpha=0.01, \quad \beta=3.2, \quad \sigma_{2}=\sigma_{3}=2.5$ and vary the values of $\lambda_{C_{0}}$ from 0.35 to 1.7 .

Case 3. We fix $\lambda_{C_{0}}=0.35, \lambda=0.2, \mu=0.4, \mu_{3}=0.6$, $\mu_{2}=0.5, \quad \theta=0.7, \quad \alpha=0.01, \quad \beta=3.2, \quad \sigma_{2}=\sigma_{3}=2.5$ and vary the values of $\lambda_{C_{1}}$ from 0.3 to 1.7 .

Case 4. We fix $\lambda_{C_{1}}=0.3, \lambda=0.2, \lambda_{C_{0}}=0.35, \mu_{3}=0.6$, $\mu_{2}=0.5, \theta=0.7, \alpha=0.01, \beta=3.2, \sigma_{2}=\sigma_{3}=2.5$ and vary the values of $\mu$ from 0.4 to 1.8 .

Case 5. We fix $\lambda_{C_{1}}=0.3, \lambda=0.2, \lambda_{C_{0}}=0.35, \mu_{3}=0.6$, $\mu=0.4, \theta=0.7, \alpha=0.01, \beta=3.2, \sigma_{2}=\sigma_{3}=2.5$ and vary the values of $\mu_{2}$ from 0.5 to 1.9 .

Case 6. We fix $\lambda_{C_{1}}=0.3, \lambda=0.2, \lambda_{C_{0}}=0.35, \mu_{2}=0.5$, $\mu=0.4, \theta=0.7, \alpha=0.01, \beta=3.2, \sigma_{2}=\sigma_{3}=2.5$ and vary the values of $\mu_{3}$ from 0.6 to 2

Table 3. Comparison of the availability models 1, 2 for $A v$ (case 1)

\begin{tabular}{c|ccc}
\hline$\lambda$ & $A v_{1}(G)$ & $A v_{1}(W)$ & $A v_{1}(L)$ \\
\hline 0.2 & 0.265172 & 0.366038 & 0.0481203 \\
0.3 & 0.235916 & 0.330661 & 0.0425285 \\
0.4 & 0.21224 & 0.301224 & 0.0380537 \\
0.5 & 0.192744 & 0.276423 & 0.0344035 \\
0.6 & 0.176443 & 0.255283 & 0.0313753 \\
0.7 & 0.162629 & 0.237073 & 0.0288263 \\
0.8 & 0.150784 & 0.221239 & 0.0266531 \\
0.9 & 0.140522 & 0.207353 & 0.0247797 \\
1.0 & 0.13155 & 0.195082 & 0.0231489
\end{tabular}




\begin{tabular}{c|ccc}
1.1 & 0.123642 & 0.184165 & 0.021717 \\
1.2 & 0.116622 & 0.174392 & 0.0204501 \\
1.3 & 0.110348 & 0.165593 & 0.0193215 \\
1.4 & 0.10471 & 0.157632 & 0.0183099 \\
1.5 & 0.0996155 & 0.150396 & 0.0173981 \\
1.6 & 0.0949906 & 0.14379 & 0.0165723 \\
\hline$\lambda$ & $A v_{2}(G)$ & $A v_{2}(W)$ & $A v_{2}(L)$ \\
\hline 0.2 & 0.21224 & 0.301224 & 0.0380537 \\
0.3 & 0.176443 & 0.255283 & 0.0313753 \\
0.4 & 0.150784 & 0.221239 & 0.0266531 \\
0.5 & 0.13155 & 0.195082 & 0.0231489 \\
0.6 & 0.116622 & 0.174392 & 0.0204501 \\
0.7 & 0.10471 & 0.157632 & 0.0183099 \\
0.8 & 0.0949906 & 0.14379 & 0.0165723 \\
0.9 & 0.0869127 & 0.132168 & 0.015134 \\
1.0 & 0.0800949 & 0.122276 & 0.0139243 \\
1.1 & 0.0742646 & 0.113755 & 0.0128929 \\
1.2 & 0.0692227 & 0.10634 & 0.0120032 \\
1.3 & 0.0648198 & 0.0998291 & 0.011228 \\
1.4 & 0.060942 & 0.0940676 & 0.0105466 \\
1.5 & 0.0575009 & 0.0889332 & 0.00994292 \\
1.6 & 0.0544268 & 0.084329 & 0.00940447 \\
\hline
\end{tabular}

Table 4. Comparison of the availability models 1,2 for Av (case 2)

\begin{tabular}{c|ccc}
\hline$\lambda_{C_{0}}$ & $A v_{1}(G)$ & $A v_{1}(W)$ & $A v_{1}(L)$ \\
\hline 0.35 & 0.265172 & 0.366038 & 0.0481203 \\
0.4 & 0.248846 & 0.346429 & 0.0440429 \\
0.5 & 0.221562 & 0.312903 & 0.0376605 \\
0.6 & 0.199671 & 0.285294 & 0.0328938 \\
0.7 & 0.181716 & 0.262162 & 0.0291982 \\
0.8 & 0.166724 & 0.2425 & 0.0262491 \\
0.9 & 0.154017 & 0.225581 & 0.0238411 \\
1.0 & 0.14311 & 0.21087 & 0.0218378 \\
1.1 & 0.133646 & 0.197959 & 0.020145 \\
1.2 & 0.125355 & 0.186538 & 0.0186958 \\
1.3 & 0.118034 & 0.176364 & 0.0174411 \\
1.4 & 0.11152 & 0.167241 & 0.0163442 \\
1.5 & 0.105688 & 0.159016 & 0.0153772 \\
1.6 & 0.100435 & 0.151563 & 0.0145181 \\
1.7 & 0.0956796 & 0.144776 & 0.01375 \\
\hline$\lambda_{C_{0}}$ & $A v_{2}(G)$ & $A v_{2}(W)$ & $A v_{2}(L)$ \\
\hline 0.35 & 0.21224 & 0.301224 & 0.0380537 \\
0.4 & 0.202015 & 0.288281 & 0.0355454 \\
0.5 & 0.18426 & 0.265468 & 0.0314052 \\
0.6 & 0.169375 & 0.246 & 0.0281289 \\
0.7 & 0.156715 & 0.229193 & 0.0254716 \\
0.8 & 0.145815 & 0.214535 & 0.023273 \\
0.9 & 0.136333 & 0.201639 & 0.0214238 \\
1.0 & 0.128009 & 0.190206 & 0.0198468 \\
1.1 & 0.120643 & 0.18 & 0.0184861 \\
1.2 & 0.114079 & 0.170833 & 0.0173 \\
1.3 & 0.108192 & 0.162555 & 0.0162569 \\
1.4 & 0.102883 & 0.155042 & 0.0153325 \\
1.5 & 0.0980705 & 0.148193 & 0.0145075 \\
1.6 & 0.0936881 & 0.141923 & 0.0137668 \\
& & &
\end{tabular}

\section{\begin{tabular}{l|lll}
1.7 & 0.0896806 & 0.136162 & 0.013098
\end{tabular}}

Table 5.Comparison of the availability models 1,2 for $A v$ (case 3)

\begin{tabular}{c|ccc}
\hline$\lambda_{C_{1}}$ & $A v_{1}(G)$ & $A v_{1}(W)$ & $A v_{1}(L)$ \\
\hline 0.3 & 0.265172 & 0.366038 & 0.0481203 \\
0.4 & 0.262796 & 0.363204 & 0.0475029 \\
0.5 & 0.260858 & 0.360888 & 0.0470029 \\
0.6 & 0.259247 & 0.35896 & 0.0465898 \\
0.7 & 0.257887 & 0.357329 & 0.0462426 \\
0.8 & 0.256724 & 0.355932 & 0.0459469 \\
0.9 & 0.255717 & 0.354722 & 0.0456918 \\
1.0 & 0.254837 & 0.353664 & 0.0454697 \\
1.1 & 0.254062 & 0.35273 & 0.0452744 \\
1.2 & 0.253374 & 0.351901 & 0.0451015 \\
1.3 & 0.252759 & 0.351159 & 0.0449472 \\
1.4 & 0.252205 & 0.350491 & 0.0448088 \\
1.5 & 0.251705 & 0.349887 & 0.0446838 \\
1.6 & 0.251251 & 0.349338 & 0.0445705 \\
1.7 & 0.250836 & 0.348837 & 0.0444672 \\
\hline$\lambda_{C_{1}}$ & $A v_{2}(G)$ & $A v_{2}(W)$ & $A v_{2}(L)$ \\
\hline 0.3 & 0.21224 & 0.301224 & 0.0380537 \\
0.4 & 0.210093 & 0.298519 & 0.0375028 \\
0.5 & 0.208281 & 0.29623 & 0.0370415 \\
0.6 & 0.206731 & 0.294268 & 0.0366497 \\
0.7 & 0.20539 & 0.292569 & 0.0363126 \\
0.8 & 0.204218 & 0.291082 & 0.0360197 \\
0.9 & 0.203186 & 0.289771 & 0.0357627 \\
1.0 & 0.202269 & 0.288605 & 0.0355355 \\
1.1 & 0.20145 & 0.287563 & 0.0353331 \\
1.2 & 0.200714 & 0.286624 & 0.0351517 \\
1.3 & 0.200048 & 0.285775 & 0.0349882 \\
1.4 & 0.199443 & 0.285004 & 0.03484 \\
1.5 & 0.198891 & 0.284299 & 0.0347051 \\
1.6 & 0.198386 & 0.283653 & 0.0345819 \\
1.7 & 0.197921 & 0.283059 & 0.0344687 \\
\hline & & &
\end{tabular}

Table 6. Comparison of the availability models 1,2 for $A v$ (case 4)

\begin{tabular}{c|ccc}
\hline$\mu$ & $A v_{1}(G)$ & $A v_{1}(W)$ & $A v_{1}(L)$ \\
\hline 0.4 & 0.265172 & 0.366038 & 0.0481203 \\
0.5 & 0.265228 & 0.366104 & 0.0481231 \\
0.6 & 0.265274 & 0.366159 & 0.0481254 \\
0.7 & 0.265313 & 0.366205 & 0.0481274 \\
0.8 & 0.265346 & 0.366245 & 0.048129 \\
0.9 & 0.265375 & 0.366279 & 0.0481305 \\
1.0 & 0.2654 & 0.366309 & 0.0481317 \\
1.1 & 0.265422 & 0.366335 & 0.0481328 \\
1.2 & 0.265442 & 0.366358 & 0.0481338 \\
1.3 & 0.265459 & 0.366379 & 0.0481347 \\
1.4 & 0.265475 & 0.366398 & 0.0481354 \\
1.5 & 0.265489 & 0.366415 & 0.0481361 \\
1.6 & 0.265502 & 0.36643 & 0.0481368 \\
1.7 & 0.265514 & 0.366444 & 0.0481374 \\
1.8 & 0.265524 & 0.366457 & 0.0481379 \\
\hline$\mu$ & $A v_{2}(G)$ & $A v_{2}(W)$ & $A v_{2}(L)$ \\
\hline
\end{tabular}




\begin{tabular}{l|ccc}
0.4 & 0.21224 & 0.301224 & 0.0380537 \\
0.5 & 0.212419 & 0.301451 & 0.0380817 \\
0.6 & 0.212574 & 0.301645 & 0.0381058 \\
0.7 & 0.212709 & 0.301815 & 0.0381268 \\
0.8 & 0.212827 & 0.301964 & 0.0381452 \\
0.9 & 0.212932 & 0.302095 & 0.0381615 \\
1.0 & 0.213025 & 0.302213 & 0.038176 \\
1.1 & 0.213109 & 0.302318 & 0.0381891 \\
1.2 & 0.213185 & 0.302413 & 0.0382008 \\
1.3 & 0.213253 & 0.3025 & 0.0382115 \\
1.4 & 0.213315 & 0.302578 & 0.0382212 \\
1.5 & 0.213373 & 0.30265 & 0.0382301 \\
1.6 & 0.213425 & 0.302716 & 0.0382383 \\
1.7 & 0.213473 & 0.302776 & 0.0382458 \\
1.8 & 0.213518 & 0.302833 & 0.0382527 \\
\hline
\end{tabular}

Table 7. Comparison of the availability models 1,2 for $A v$ (case 5)

\begin{tabular}{c|ccc}
\hline$\mu_{2}$ & $A v_{1}(G)$ & $A v_{1}(W)$ & $A v_{1}(L)$ \\
\hline 0.5 & 0.265172 & 0.366038 & 0.0481203 \\
0.6 & 0.276458 & 0.3794 & 0.0468366 \\
0.7 & 0.285126 & 0.389558 & 0.0454952 \\
0.8 & 0.291993 & 0.397541 & 0.0440994 \\
0.9 & 0.297566 & 0.40398 & 0.0426532 \\
1.0 & 0.302181 & 0.409283 & 0.0411613 \\
1.1 & 0.306064 & 0.413726 & 0.0396294 \\
1.2 & 0.309377 & 0.417504 & 0.0380638 \\
1.3 & 0.312237 & 0.420754 & 0.0364715 \\
1.4 & 0.314731 & 0.423581 & 0.0348598 \\
1.5 & 0.316924 & 0.426061 & 0.0332366 \\
1.6 & 0.318869 & 0.428256 & 0.0316099 \\
1.7 & 0.320605 & 0.430211 & 0.0299878 \\
1.8 & 0.322164 & 0.431964 & 0.0283785 \\
1.9 & 0.323572 & 0.433545 & 0.0267895 \\
\hline$\mu_{2}$ & $A v_{2}(G)$ & $A v_{2}(W)$ & $A v_{2}(L)$ \\
\hline 0.5 & 0.21224 & 0.301224 & 0.0380537 \\
0.6 & 0.227301 & 0.320035 & 0.0364515 \\
0.7 & 0.239437 & 0.334976 & 0.0348308 \\
0.8 & 0.249425 & 0.347131 & 0.0331994 \\
0.9 & 0.25779 & 0.357212 & 0.0315654 \\
1.0 & 0.264896 & 0.365709 & 0.029937 \\
1.1 & 0.271008 & 0.372967 & 0.0283223 \\
1.2 & 0.276322 & 0.379239 & 0.026729 \\
1.3 & 0.280983 & 0.384714 & 0.0251645 \\
1.4 & 0.285106 & 0.389534 & 0.0236355 \\
1.5 & 0.288778 & 0.39381 & 0.0221483 \\
1.6 & 0.292069 & 0.397629 & 0.0207082 \\
1.7 & 0.295036 & 0.401061 & 0.0193199 \\
1.8 & 0.297725 & 0.404162 & 0.0179872 \\
1.9 & 0.300172 & 0.406977 & 0.0167131 \\
\hline & & &
\end{tabular}

Table 8.Comparison of the availability models 1, 2 for $A v$ (case 6)

\begin{tabular}{c|ccc}
\hline$\mu_{3}$ & $A v_{1}(G)$ & $A v_{1}(W)$ & $A v_{1}(L)$ \\
\hline 0.6 & 0.265172 & 0.366038 & 0.0481203 \\
0.7 & 0.285126 & 0.389558 & 0.0448591 \\
0.8 & 0.302181 & 0.409283 & 0.0417332
\end{tabular}

\begin{tabular}{c|ccc}
0.9 & 0.316924 & 0.426061 & 0.0387491 \\
1.0 & 0.329797 & 0.440509 & 0.0359112 \\
1.1 & 0.341134 & 0.453079 & 0.0332223 \\
1.2 & 0.351195 & 0.464115 & 0.0306831 \\
1.3 & 0.360183 & 0.473882 & 0.0282933 \\
1.4 & 0.368261 & 0.482587 & 0.0260508 \\
1.5 & 0.375561 & 0.490394 & 0.0239528 \\
1.6 & 0.382191 & 0.497436 & 0.021995 \\
1.7 & 0.388238 & 0.503819 & 0.0201728 \\
1.8 & 0.393775 & 0.509632 & 0.0184807 \\
1.9 & 0.398866 & 0.514948 & 0.0169129 \\
2 & 0.403561 & 0.519829 & 0.0154631 \\
\hline$\mu_{3}$ & $A v_{2}(G)$ & $A v_{2}(W)$ & $A v_{2}(L)$ \\
\hline 0.6 & 0.21224 & 0.301224 & 0.038053 \\
0.7 & 0.224769 & 0.316894 & 0.035994 \\
0.8 & 0.235182 & 0.329759 & 0.033963 \\
0.9 & 0.243972 & 0.340511 & 0.031969 \\
1.0 & 0.251493 & 0.34963 & 0.030021 \\
1.1 & 0.257999 & 0.357464 & 0.028127 \\
1.2 & 0.263684 & 0.364265 & 0.026294 \\
1.3 & 0.268694 & 0.370225 & 0.024527 \\
1.4 & 0.273143 & 0.375491 & 0.022831 \\
1.5 & 0.277119 & 0.380177 & 0.021211 \\
1.6 & 0.280694 & 0.384375 & 0.019668 \\
1.7 & 0.283926 & 0.388157 & 0.018205 \\
1.8 & 0.286862 & 0.391581 & 0.016821 \\
1.9 & 0.289541 & 0.394697 & 0.015518 \\
2 & 0.291995 & 0.397544 & 0.014294 \\
\hline & & & \\
\hline
\end{tabular}

\section{$4.2 \mathrm{cost} / \mathrm{benefit}$ ratio comparisons}

Let $b_{i}=C_{i} / A v_{i}$ where $C_{i}$ be the cost of the configuration $i$, for $i=1,2$ which are listed in Table 2 . We Compare $b_{i}, i=1,2$ in six cases as follow:

Table 9. Comparison of the cost/benefit models 1, 2 for (case 1)

\begin{tabular}{c|ccc}
\hline$\lambda$ & $b_{1}(G)$ & $b_{1}(W)$ & $b_{1}(L)$ \\
\hline 0.2 & $1.50845 \times 10^{8}$ & $1.09278 \times 10^{8}$ & $8.31249 \times 10^{8}$ \\
0.3 & $1.69552 \times 10^{8}$ & $1.2097 \times 10^{8}$ & $9.40546 \times 10^{8}$ \\
0.4 & $1.88466 \times 10^{8}$ & $1.32791 \times 10^{8}$ & $1.05114 \times 10^{9}$ \\
0.5 & $2.07529 \times 10^{8}$ & $1.44706 \times 10^{8}$ & $1.16267 \times 10^{9}$ \\
0.6 & $2.26702 \times 10^{8}$ & $1.56689 \times 10^{8}$ & $1.27489 \times 10^{9}$ \\
0.7 & $2.45959 \times 10^{8}$ & $1.68724 \times 10^{8}$ & $1.38762 \times 10^{9}$ \\
0.8 & $2.6528 \times 10^{8}$ & $1.808 \times 10^{8}$ & $1.50076 \times 10^{9}$ \\
0.9 & $2.84652 \times 10^{8}$ & $1.92908 \times 10^{8}$ & $1.61423 \times 10^{9}$ \\
1.0 & $3.04066 \times 10^{8}$ & $2.05041 \times 10^{8}$ & $1.72795 \times 10^{9}$ \\
1.1 & $3.23514 \times 10^{8}$ & $2.17196 \times 10^{8}$ & $1.84188 \times 10^{9}$ \\
1.2 & $3.4299 \times 10^{8}$ & $2.29369 \times 10^{8}$ & $1.95598 \times 10^{9}$ \\
1.3 & $3.62489 \times 10^{8}$ & $2.41556 \times 10^{8}$ & $2.07024 \times 10^{9}$ \\
1.4 & $3.82008 \times 10^{8}$ & $2.53755 \times 10^{8}$ & $2.18462 \times 10^{9}$ \\
1.5 & $4.01544 \times 10^{8}$ & $2.65965 \times 10^{8}$ & $2.2991 \times 10^{9}$ \\
1.6 & $4.21094 \times 10^{8}$ & $2.78184 \times 10^{8}$ & $2.41367 \times 10^{9}$ \\
\hline$\lambda$ & $b_{2}(G)$ & $b_{2}(W)$ & $b_{2}(L)$ \\
\hline 0.2 & $1.64908 \times 10^{8}$ & $1.16192 \times 10^{8}$ & $9.19752 \times 10^{8}$ \\
0.3 & $1.98365 \times 10^{8}$ & $1.37103 \times 10^{8}$ & $1.11553 \times 10^{9}$ \\
0.4 & $2.3212 \times 10^{8}$ & $1.582 \times 10^{8}$ & $1.31317 \times 10^{9}$ \\
0.5 & $2.66058 \times 10^{8}$ & $1.79411 \times 10^{8}$ & $1.51195 \times 10^{9}$ \\
0.6 & $3.00116 \times 10^{8}$ & $2.00698 \times 10^{8}$ & $1.71149 \times 10^{9}$ \\
& & &
\end{tabular}




\begin{tabular}{c|ccc}
0.7 & $3.34257 \times 10^{8}$ & $2.22036 \times 10^{8}$ & $1.91154 \times 10^{9}$ \\
0.8 & $3.68458 \times 10^{8}$ & $2.43411 \times 10^{8}$ & $2.11196 \times 10^{9}$ \\
0.9 & $4.02703 \times 10^{8}$ & $2.64814 \times 10^{8}$ & $2.31267 \times 10^{9}$ \\
1.0 & $4.36982 \times 10^{8}$ & $2.86239 \times 10^{8}$ & $2.51358 \times 10^{9}$ \\
1.1 & $4.71288 \times 10^{8}$ & $3.0768 \times 10^{8}$ & $2.71467 \times 10^{9}$ \\
1.2 & $5.05614 \times 10^{8}$ & $3.29134 \times 10^{8}$ & $2.91588 \times 10^{9}$ \\
1.3 & $5.39958 \times 10^{8}$ & $3.50599 \times 10^{8}$ & $3.1172 \times 10^{9}$ \\
1.4 & $5.74316 \times 10^{8}$ & $3.72073 \times 10^{8}$ & $3.31861 \times 10^{9}$ \\
1.5 & $6.08686 \times 10^{8}$ & $3.93554 \times 10^{8}$ & $3.52009 \times 10^{9}$ \\
1.6 & $6.43066 \times 10^{8}$ & $4.15041 \times 10^{8}$ & $3.72164 \times 10^{9}$ \\
\hline
\end{tabular}

Table 10. Comparison of the cost/benefit models 1,2 for (case 2)

\begin{tabular}{|c|c|c|c|}
\hline$\lambda_{C_{0}}$ & $b_{1}(G)$ & $b_{1}(W)$ & $b_{1}(L)$ \\
\hline 0.35 & $1.50845 \times 10^{8}$ & $1.09278 \times 10^{8}$ & $8.31249 \times 10^{8}$ \\
\hline 0.4 & $1.60742 \times 10^{8}$ & $1.15464 \times 10^{8}$ & $9.08206 \times 10^{8}$ \\
\hline 0.5 & $1.80536 \times 10^{8}$ & $1.27835 \times 10^{8}$ & $1.06212 \times 10^{9}$ \\
\hline 0.6 & $2.0033 \times 10^{8}$ & $1.40206 \times 10^{8}$ & $1.21603 \times 10^{9}$ \\
\hline 0.7 & $2.20124 \times 10^{8}$ & $1.52577 \times 10^{8}$ & $1.36995 \times 10^{9}$ \\
\hline 0.8 & $2.39918 \times 10^{8}$ & $1.64948 \times 10^{8}$ & $1.52386 \times 10^{9}$ \\
\hline 0.9 & $2.59711 \times 10^{8}$ & $1.7732 \times 10^{8}$ & $1.67778 \times 10^{9}$ \\
\hline 1.0 & $2.79505 \times 10^{8}$ & $1.89691 \times 10^{8}$ & $1.83169 \times 10^{9}$ \\
\hline 1.1 & $2.99299 \times 10^{8}$ & $2.02062 \times 10^{8}$ & $1.9856 \times 10^{9}$ \\
\hline 1.2 & $3.19093 \times 10^{8}$ & $2.14433 \times 10^{8}$ & $2.13952 \times 10^{9}$ \\
\hline 1.3 & $3.38887 \times 10^{8}$ & $2.26804 \times 10^{8}$ & $2.29343 \times 10^{9}$ \\
\hline 1.4 & $3.5868 \times 10^{8}$ & $2.39175 \times 10^{8}$ & $2.44734 \times 10^{9}$ \\
\hline 1.5 & $3.78474 \times 10^{8}$ & $2.51546 \times 10^{8}$ & $2.60126 \times 10^{9}$ \\
\hline 1.6 & $3.98268 \times 10^{8}$ & $2.63918 \times 10^{8}$ & $2.75517 \times 10^{9}$ \\
\hline 1.7 & $4.18062 \times 10^{8}$ & $2.76289 \times 10^{8}$ & $2.90909 \times 10^{9}$ \\
\hline$\lambda_{C_{0}}$ & $b_{2}(G)$ & $b_{2}(W)$ & $b_{2}(L)$ \\
\hline 0.35 & $1.64908 \times 10^{8}$ & $1.16192 \times 10^{8}$ & $9.19752 \times 10^{8}$ \\
\hline 0.4 & $1.73255 \times 10^{8}$ & $1.21409 \times 10^{8}$ & $9.84656 \times 10^{8}$ \\
\hline 0.5 & $1.89949 \times 10^{8}$ & $1.31843 \times 10^{8}$ & $1.11446 \times 10^{9}$ \\
\hline 0.6 & $2.06642 \times 10^{8}$ & $1.42276 \times 10^{8}$ & $1.24427 \times 10^{9}$ \\
\hline 0.7 & $2.23336 \times 10^{8}$ & $1.5271 \times 10^{8}$ & $1.37408 \times 10^{9}$ \\
\hline 0.8 & $2.4003 \times 10^{8}$ & $1.63144 \times 10^{8}$ & $1.50389 \times 10^{9}$ \\
\hline 0.9 & $2.56724 \times 10^{8}$ & $1.73577 \times 10^{8}$ & $1.6337 \times 10^{9}$ \\
\hline 1.0 & $2.73417 \times 10^{8}$ & $1.84011 \times 10^{8}$ & $1.76351 \times 10^{9}$ \\
\hline 1.1 & $2.90111 \times 10^{8}$ & $1.94444 \times 10^{8}$ & $1.89331 \times 10^{9}$ \\
\hline 1.2 & $3.06805 \times 10^{8}$ & $2.04878 \times 10^{8}$ & $2.02312 \times 10^{9}$ \\
\hline 1.3 & $3.23499 \times 10^{8}$ & $2.15312 \times 10^{8}$ & $2.15293 \times 10^{9}$ \\
\hline 1.4 & $3.40192 \times 10^{8}$ & $2.25745 \times 10^{8}$ & $2.28274 \times 10^{9}$ \\
\hline 1.5 & $3.56886 \times 10^{8}$ & $2.36179 \times 10^{8}$ & $2.41255 \times 10^{9}$ \\
\hline 1.6 & $3.7358 \times 10^{8}$ & $2.46612 \times 10^{8}$ & $2.54236 \times 10^{9}$ \\
\hline 1.7 & $3.90274 \times 10^{8}$ & $2.57046 \times 10^{8}$ & $2.67216 \times 10^{9}$ \\
\hline
\end{tabular}

Table 11. Comparison of the cost/benefit models 1,2 for (case 3)

\begin{tabular}{c|ccc}
\hline$\lambda_{C_{1}}$ & $b_{1}(G)$ & $b_{1}(W)$ & $b_{1}(L)$ \\
\hline 0.3 & $1.50845 \times 10^{8}$ & $1.09278 \times 10^{8}$ & $8.31249 \times 10^{8}$ \\
0.4 & $1.52209 \times 10^{8}$ & $1.10131 \times 10^{8}$ & $8.42054 \times 10^{8}$ \\
0.5 & $1.5334 \times 10^{8}$ & $1.10838 \times 10^{8}$ & $8.51011 \times 10^{8}$ \\
0.6 & $1.54293 \times 10^{8}$ & $1.11433 \times 10^{8}$ & $8.58558 \times 10^{8}$ \\
0.7 & $1.55107 \times 10^{8}$ & $1.11942 \times 10^{8}$ & $8.65003 \times 10^{8}$ \\
0.8 & $1.5581 \times 10^{8}$ & $1.12381 \times 10^{8}$ & $8.70571 \times 10^{8}$
\end{tabular}

\begin{tabular}{c|ccc}
0.9 & $1.56423 \times 10^{8}$ & $1.12764 \times 10^{8}$ & $8.7543 \times 10^{8}$ \\
1.0 & $1.56963 \times 10^{8}$ & $1.13102 \times 10^{8}$ & $8.79707 \times 10^{8}$ \\
1.1 & $1.57442 \times 10^{8}$ & $1.13401 \times 10^{8}$ & $8.83501 \times 10^{8}$ \\
1.2 & $1.5787 \times 10^{8}$ & $1.13668 \times 10^{8}$ & $8.86888 \times 10^{8}$ \\
1.3 & $1.58254 \times 10^{8}$ & $1.13909 \times 10^{8}$ & $8.89932 \times 10^{8}$ \\
1.4 & $1.58601 \times 10^{8}$ & $1.14126 \times 10^{8}$ & $8.92682 \times 10^{8}$ \\
1.5 & $1.58916 \times 10^{8}$ & $1.14323 \times 10^{8}$ & $8.95179 \times 10^{8}$ \\
1.6 & $1.59204 \times 10^{8}$ & $1.14502 \times 10^{8}$ & $8.97455 \times 10^{8}$ \\
1.7 & $1.59467 \times 10^{8}$ & $1.14667 \times 10^{8}$ & $8.9954 \times 10^{8}$ \\
\hline$\lambda_{C_{1}}$ & $b_{2}(G)$ & $b_{2}(W)$ & $b 2(L)$ \\
\hline 0.3 & $1.64908 \times 10^{8}$ & $1.16192 \times 10^{8}$ & $9.19752 \times 10^{8}$ \\
0.4 & $1.66593 \times 10^{8}$ & $1.17246 \times 10^{8}$ & $9.33264 \times 10^{8}$ \\
0.5 & $1.68042 \times 10^{8}$ & $1.18152 \times 10^{8}$ & $9.44886 \times 10^{8}$ \\
0.6 & $1.69302 \times 10^{8}$ & $1.18939 \times 10^{8}$ & $9.54988 \times 10^{8}$ \\
0.7 & $1.70408 \times 10^{8}$ & $1.1963 \times 10^{8}$ & $9.63852 \times 10^{8}$ \\
0.8 & $1.71385 \times 10^{8}$ & $1.20241 \times 10^{8}$ & $9.7169 \times 10^{8}$ \\
0.9 & $1.72256 \times 10^{8}$ & $1.20785 \times 10^{8}$ & $9.78672 \times 10^{8}$ \\
1.0 & $1.73037 \times 10^{8}$ & $1.21273 \times 10^{8}$ & $9.84931 \times 10^{8}$ \\
1.1 & $1.7374 \times 10^{8}$ & $1.21713 \times 10^{8}$ & $9.90572 \times 10^{8}$ \\
1.2 & $1.74378 \times 10^{8}$ & $1.22111 \times 10^{8}$ & $9.95684 \times 10^{8}$ \\
1.3 & $1.74958 \times 10^{8}$ & $1.22474 \times 10^{8}$ & $1.00034 \times 10^{9}$ \\
1.4 & $1.75489 \times 10^{8}$ & $1.22805 \times 10^{8}$ & $1.00459 \times 10^{9}$ \\
1.5 & $1.75976 \times 10^{8}$ & $1.2311 \times 10^{8}$ & $1.0085 \times 10^{9}$ \\
1.6 & $1.76424 \times 10^{8}$ & $1.2339 \times 10^{8}$ & $1.01209 \times 10^{9}$ \\
1.7 & $1.76838 \times 10^{8}$ & $1.23649 \times 10^{8}$ & $1.01541 \times 10^{9}$ \\
\hline & & & \\
\hline
\end{tabular}

Table 12. Comparison of the cost/benefit models 1,2 for $A v$ (case 4)

\begin{tabular}{c|ccc}
\hline$\mu$ & $b_{1}(G)$ & $b_{1}(W)$ & $b_{1}(L)$ \\
\hline 0.4 & $1.50845 \times 10^{8}$ & $1.09278 \times 10^{8}$ & $8.31249 \times 10^{8}$ \\
0.5 & $1.50814 \times 10^{8}$ & $1.09259 \times 10^{8}$ & $8.31201 \times 10^{8}$ \\
0.6 & $1.50787 \times 10^{8}$ & $1.09242 \times 10^{8}$ & $8.31162 \times 10^{8}$ \\
0.7 & $1.50765 \times 10^{8}$ & $1.09228 \times 10^{8}$ & $8.31128 \times 10^{8}$ \\
0.8 & $1.50746 \times 10^{8}$ & $1.09217 \times 10^{8}$ & $8.31099 \times 10^{8}$ \\
0.9 & $1.5073 \times 10^{8}$ & $1.09206 \times 10^{8}$ & $8.31075 \times 10^{8}$ \\
1.0 & $1.50716 \times 10^{8}$ & $1.09197 \times 10^{8}$ & $8.31053 \times 10^{8}$ \\
1.1 & $1.50703 \times 10^{8}$ & $1.0919 \times 10^{8}$ & $8.31034 \times 10^{8}$ \\
1.2 & $1.50692 \times 10^{8}$ & $1.09183 \times 10^{8}$ & $8.31017 \times 10^{8}$ \\
1.3 & $1.50682 \times 10^{8}$ & $1.09176 \times 10^{8}$ & $8.31002 \times 10^{8}$ \\
1.4 & $1.50673 \times 10^{8}$ & $1.09171 \times 10^{8}$ & $8.30989 \times 10^{8}$ \\
1.5 & $1.50665 \times 10^{8}$ & $1.09166 \times 10^{8}$ & $8.30976 \times 10^{8}$ \\
1.6 & $1.50658 \times 10^{8}$ & $1.09161 \times 10^{8}$ & $8.30965 \times 10^{8}$ \\
1.7 & $1.50651 \times 10^{8}$ & $1.09157 \times 10^{8}$ & $8.30955 \times 10^{8}$ \\
1.8 & $1.50645 \times 10^{8}$ & $1.09153 \times 10^{8}$ & $8.30946 \times 10^{8}$ \\
\hline$\mu$ & $b_{2}(G)$ & $b_{2}(W)$ & $b_{2}(L)$ \\
\hline 0.4 & $1.64908 \times 10^{8}$ & $1.16192 \times 10^{8}$ & $9.19752 \times 10^{8}$ \\
0.5 & $1.64768 \times 10^{8}$ & $1.16105 \times 10^{8}$ & $9.19076 \times 10^{8}$ \\
0.6 & $1.64648 \times 10^{8}$ & $1.1603 \times 10^{8}$ & $9.18495 \times 10^{8}$ \\
0.7 & $1.64544 \times 10^{8}$ & $1.15965 \times 10^{8}$ & $9.1799 \times 10^{8}$ \\
0.8 & $1.64453 \times 10^{8}$ & $1.15908 \times 10^{8}$ & $9.17546 \times 10^{8}$ \\
0.9 & $1.64372 \times 10^{8}$ & $1.15857 \times 10^{8}$ & $9.17154 \times 10^{8}$ \\
1.0 & $1.643 \times 10^{8}$ & $1.15812 \times 10^{8}$ & $9.16805 \times 10^{8}$ \\
1.1 & $1.64235 \times 10^{8}$ & $1.15772 \times 10^{8}$ & $9.16492 \times 10^{8}$ \\
1.2 & $1.64177 \times 10^{8}$ & $1.15736 \times 10^{8}$ & $9.1621 \times 10^{8}$ \\
1.3 & $1.64124 \times 10^{8}$ & $1.15703 \times 10^{8}$ & $9.15954 \times 10^{8}$ \\
1.4 & $1.64076 \times 10^{8}$ & $1.15673 \times 10^{8}$ & $9.15722 \times 10^{8}$
\end{tabular}




\begin{tabular}{c|ccc}
1.5 & $1.64032 \times 10^{8}$ & $1.15645 \times 10^{8}$ & $9.15509 \times 10^{8}$ \\
1.6 & $1.63992 \times 10^{8}$ & $1.1562 \times 10^{8}$ & $9.15314 \times 10^{8}$ \\
1.7 & $1.63955 \times 10^{8}$ & $1.15597 \times 10^{8}$ & $9.15134 \times 10^{8}$ \\
1.8 & $1.63921 \times 10^{8}$ & $1.15575 \times 10^{8}$ & $9.14968 \times 10^{8}$ \\
\hline
\end{tabular}

Table 13. Comparison of the cost/benefit models 1,2 for $A v$ (case 5)

\begin{tabular}{c|ccc}
\hline$\mu_{2}$ & $b_{1}(G)$ & $b_{1}(W)$ & $b_{1}(L)$ \\
\hline 0.5 & $1.50845 \times 10^{8}$ & $1.09278 \times 10^{8}$ & $8.31249 \times 10^{8}$ \\
0.6 & $1.44687 \times 10^{8}$ & $1.0543 \times 10^{8}$ & $8.54033 \times 10^{8}$ \\
0.7 & $1.40289 \times 10^{8}$ & $1.0268 \times 10^{8}$ & $8.79213 \times 10^{8}$ \\
0.8 & $1.3699 \times 10^{8}$ & $1.00619 \times 10^{8}$ & $9.07042 \times 10^{8}$ \\
0.9 & $1.34424 \times 10^{8}$ & $9.90149 \times 10^{7}$ & $9.37797 \times 10^{8}$ \\
1.0 & $1.32371 \times 10^{8}$ & $9.7732 \times 10^{7}$ & $9.71787 \times 10^{8}$ \\
1.1 & $1.30692 \times 10^{8}$ & $9.66823 \times 10^{7}$ & $1.00935 \times 10^{9}$ \\
1.2 & $1.29292 \times 10^{8}$ & $9.58076 \times 10^{7}$ & $1.05087 \times 10^{9}$ \\
1.3 & $1.28108 \times 10^{8}$ & $9.50674 \times 10^{7}$ & $1.09675 \times 10^{9}$ \\
1.4 & $1.27093 \times 10^{8}$ & $9.4433 \times 10^{7}$ & $1.14745 \times 10^{9}$ \\
1.5 & $1.26213 \times 10^{8}$ & $9.38832 \times 10^{7}$ & $1.20349 \times 10^{9}$ \\
1.6 & $1.25443 \times 10^{8}$ & $9.34021 \times 10^{7}$ & $1.26543 \times 10^{9}$ \\
1.7 & $1.24764 \times 10^{8}$ & $9.29776 \times 10^{7}$ & $1.33387 \times 10^{9}$ \\
1.8 & $1.2416 \times 10^{8}$ & $9.26002 \times 10^{7}$ & $1.40952 \times 10^{9}$ \\
1.9 & $1.2362 \times 10^{8}$ & $9.22626 \times 10^{7}$ & $1.49312 \times 10^{9}$ \\
\hline$\mu_{2}$ & $b_{2}(G)$ & $b_{2}(W)$ & $b_{2}(L)$ \\
\hline 0.5 & $1.64908 \times 10^{8}$ & $1.16192 \times 10^{8}$ & $9.19752 \times 10^{8}$ \\
0.6 & $1.53981 \times 10^{8}$ & $1.09363 \times 10^{8}$ & $9.60179 \times 10^{8}$ \\
0.7 & $1.46176 \times 10^{8}$ & $1.04485 \times 10^{8}$ & $1.00486 \times 10^{9}$ \\
0.8 & $1.40322 \times 10^{8}$ & $1.00827 \times 10^{8}$ & $1.05424 \times 10^{9}$ \\
0.9 & $1.3577 \times 10^{8}$ & $9.7981 \times 10^{7}$ & $1.10881 \times 10^{9}$ \\
1.0 & $1.32127 \times 10^{8}$ & $9.57046 \times 10^{7}$ & $1.16912 \times 10^{9}$ \\
1.1 & $1.29147 \times 10^{8}$ & $9.38421 \times 10^{7}$ & $1.23577 \times 10^{9}$ \\
1.2 & $1.26664 \times 10^{8}$ & $9.229 \times 10^{7}$ & $1.30944 \times 10^{9}$ \\
1.3 & $1.24563 \times 10^{8}$ & $9.09767 \times 10^{7}$ & $1.39085 \times 10^{9}$ \\
1.4 & $1.22762 \times 10^{8}$ & $8.98509 \times 10^{7}$ & $1.48082 \times 10^{9}$ \\
1.5 & $1.21201 \times 10^{8}$ & $8.88753 \times 10^{7}$ & $1.58026 \times 10^{9}$ \\
1.6 & $1.19835 \times 10^{8}$ & $8.80217 \times 10^{7}$ & $1.69015 \times 10^{9}$ \\
1.7 & $1.1863 \times 10^{8}$ & $8.72685 \times 10^{7}$ & $1.8116 \times 10^{9}$ \\
1.8 & $1.17558 \times 10^{8}$ & $8.65989 \times 10^{7}$ & $1.94583 \times 10^{9}$ \\
1.9 & $1.166 \times 10^{8}$ & $8.59999 \times 10^{7}$ & $2.09417 \times 10^{9}$ \\
& & &
\end{tabular}

Table 14. Comparison of the cost/benefit models 1, 2 for $A v$ (case 6)

\begin{tabular}{c|ccc}
\hline$\mu_{3}$ & $b_{1}(G)$ & $b_{1}(W)$ & $b_{1}(L)$ \\
\hline 0.6 & $1.50845 \times 10^{8}$ & $1.09278 \times 10^{8}$ & $8.31249 \times 10^{8}$ \\
0.7 & $1.40289 \times 10^{8}$ & $1.0268 \times 10^{8}$ & $8.91682 \times 10^{8}$ \\
0.8 & $1.32371 \times 10^{8}$ & $9.7732 \times 10^{7}$ & $9.5847 \times 10^{8}$ \\
0.9 & $1.26213 \times 10^{8}$ & $9.38832 \times 10^{7}$ & $1.03228 \times 10^{9}$ \\
1.0 & $1.21287 \times 10^{8}$ & $9.08041 \times 10^{7}$ & $1.11386 \times 10^{9}$ \\
1.1 & $1.17256 \times 10^{8}$ & $8.82849 \times 10^{7}$ & $1.20401 \times 10^{9}$ \\
1.2 & $1.13897 \times 10^{8}$ & $8.61856 \times 10^{7}$ & $1.30365 \times 10^{9}$ \\
1.3 & $1.11055 \times 10^{8}$ & $8.44092 \times 10^{7}$ & $1.41376 \times 10^{9}$ \\
1.4 & $1.08619 \times 10^{8}$ & $8.28866 \times 10^{7}$ & $1.53546 \times 10^{9}$ \\
1.5 & $1.06507 \times 10^{8}$ & $8.1567 \times 10^{7}$ & $1.66995 \times 10^{9}$ \\
1.6 & $1.0466 \times 10^{8}$ & $8.04124 \times 10^{7}$ & $1.81859 \times 10^{9}$ \\
1.7 & $1.0303 \times 10^{8}$ & $7.93936 \times 10^{7}$ & $1.98287 \times 10^{9}$ \\
1.8 & $1.01581 \times 10^{8}$ & $7.8488 \times 10^{7}$ & $2.16442 \times 10^{9}$ \\
1.9 & $1.00284 \times 10^{8}$ & $7.76777 \times 10^{7}$ & $2.36506 \times 10^{9}$
\end{tabular}

\begin{tabular}{c|ccc}
2 & $9.91175 \times 10^{7}$ & $7.69485 \times 10^{7}$ & $2.5868 \times 10^{9}$ \\
\hline$\mu_{3}$ & $b_{2}(G)$ & $b_{2}(W)$ & $b_{2}(L)$ \\
\hline 0.6 & $1.64908 \times 10^{8}$ & $1.16192 \times 10^{8}$ & $9.19752 \times 10^{8}$ \\
0.7 & $1.55715 \times 10^{8}$ & $1.10447 \times 10^{8}$ & $9.72374 \times 10^{8}$ \\
0.8 & $1.48821 \times 10^{8}$ & $1.06138 \times 10^{8}$ & $1.03053 \times 10^{9}$ \\
0.9 & $1.43459 \times 10^{8}$ & $1.02787 \times 10^{8}$ & $1.0948 \times 10^{9}$ \\
1.0 & $1.39169 \times 10^{8}$ & $1.00106 \times 10^{8}$ & $1.16584 \times 10^{9}$ \\
1.1 & $1.35659 \times 10^{8}$ & $9.7912 \times 10^{7}$ & $1.24434 \times 10^{9}$ \\
1.2 & $1.32734 \times 10^{8}$ & $9.6084 \times 10^{7}$ & $1.3311 \times 10^{9}$ \\
1.3 & $1.3026 \times 10^{8}$ & $9.45372 \times 10^{7}$ & $1.42699 \times 10^{9}$ \\
1.4 & $1.28138 \times 10^{8}$ & $9.32114 \times 10^{7}$ & $1.53295 \times 10^{9}$ \\
1.5 & $1.263 \times 10^{8}$ & $9.20623 \times 10^{7}$ & $1.65007 \times 10^{9}$ \\
1.6 & $1.24691 \times 10^{8}$ & $9.10569 \times 10^{7}$ & $1.7795 \times 10^{9}$ \\
1.7 & $1.23272 \times 10^{8}$ & $9.01698 \times 10^{7}$ & $1.92254 \times 10^{9}$ \\
1.8 & $1.2201 \times 10^{8}$ & $8.93812 \times 10^{7}$ & $2.08063 \times 10^{9}$ \\
1.9 & $1.20881 \times 10^{8}$ & $8.86757 \times 10^{7}$ & $2.25534 \times 10^{9}$ \\
2 & $1.19865 \times 10^{8}$ & $8.80407 \times 10^{7}$ & $2.44843 \times 10^{9}$ \\
\hline
\end{tabular}

Numerical results of the $b_{i}$ for configuration $i(i=1,2)$ are calculated in Tables 9-14 for six cases, respectively. From these Tables 9-14, we deduce the optimal configuration as follow:

cases $(1,3,4)$

The optimal configuration is configuration 1 , for three different repair time distributions: Gamma, Weibull, and lognormal.

case 2

1) Gamma distribution

$\lambda_{C_{0}} \in\{0.35, \ldots ., 0.8\}$ the optimal configuration is configuration $1, \quad \lambda_{C_{0}} \in\{0.9, \ldots ., 1.7\} \quad$ the optimal configuration is configuration 2 .

2) Weibull distribution and lognormal distribution

$\lambda_{C_{0}} \in\{0.35, \ldots ., 0.7\}$ the optimal configuration is configuration $1, \quad \lambda_{C_{0}} \in\{0.8, \ldots ., 1.7\}$ the optimal configuration is configuration 2 .

case 5

1) Gamma distribution

$\mu_{2} \in\{0.5, \ldots ., 0.9\}$ the optimal configuration is configuration $1, \mu_{2} \in\{1, \ldots ., 1.9\}$ the optimal configuration is configuration 2 .

2) Weibull distribution

$\mu_{2} \in\{0.5, \ldots ., 0.8\}$ the optimal configuration is configuration $1, \mu_{2} \in\{0.9, \ldots ., 1.9\}$ the optimal configuration is configuration 2 .

3) lognormal distribution

$\mu_{2} \in\{0.5, \ldots ., 1.9\}$ the optimal configuration is configuration 1 .

case 6

1) Gamma distribution and Weibull distribution

$\mu_{3} \in\{0.6, \ldots ., 2\}$ the optimal configuration is configuration

2) lognormal distribution

$\mu_{3} \in\{0.6, \ldots ., 1.3\}$ the optimal configuration is configuration $1, \mu_{3} \in\{1.4, \ldots ., 2\}$ the optimal configuration is configuration 2 .

\section{CONCLUSIONS}

In this paper we have first utilized the supplementary variable technique to develop the steady-state availability, $A v$, of two 
different series system configurations with warm standby components,

switching failures, two types of failures and general repair times. Next, for each configuration, we present the explicit expressions for the $A v$ for three various repair time distributions such as Gamma (G), Weibull (W) and Lognormal (L). Finally, we rank two configurations based on the $\mathrm{Av}$ and the $\cos t / A v$ for three various repair time distributions.

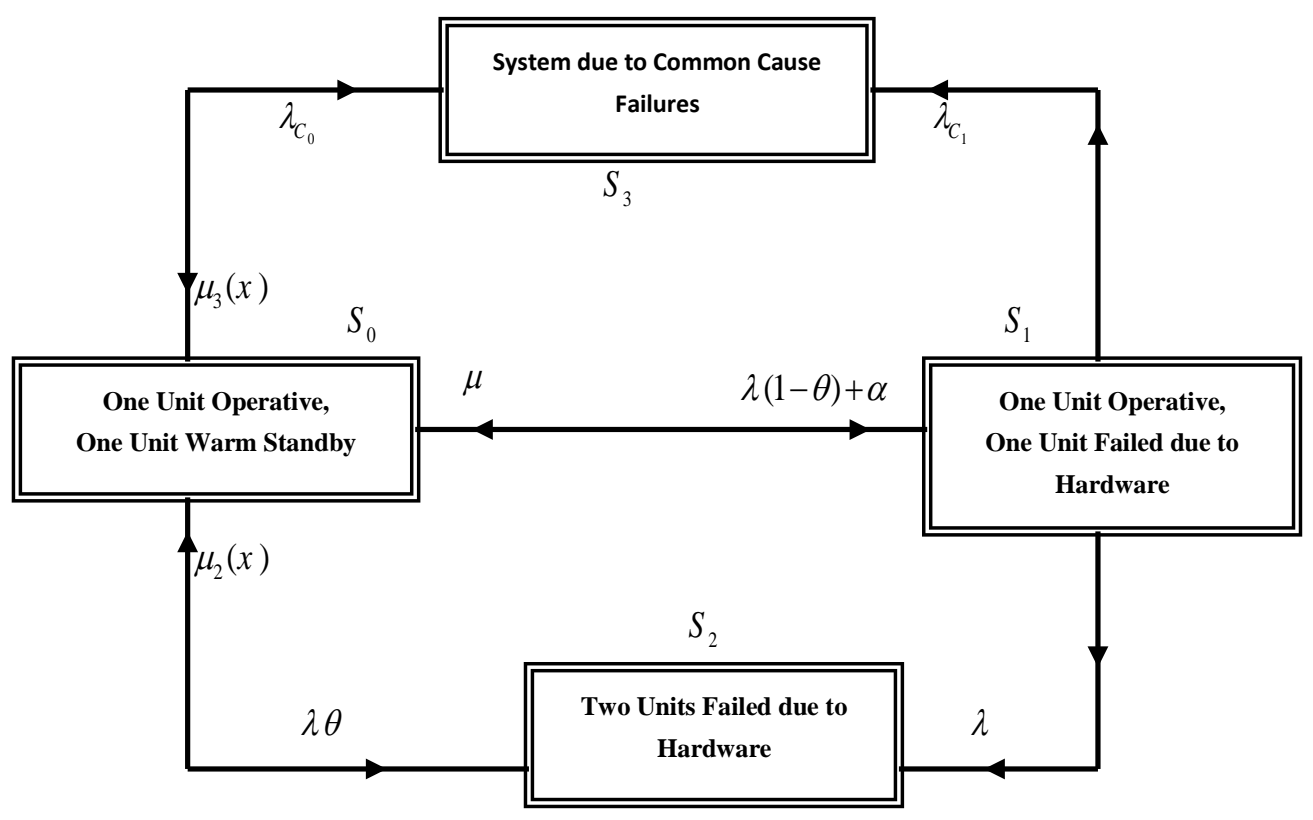

Fig 1: State-space diagram for configuration 1.

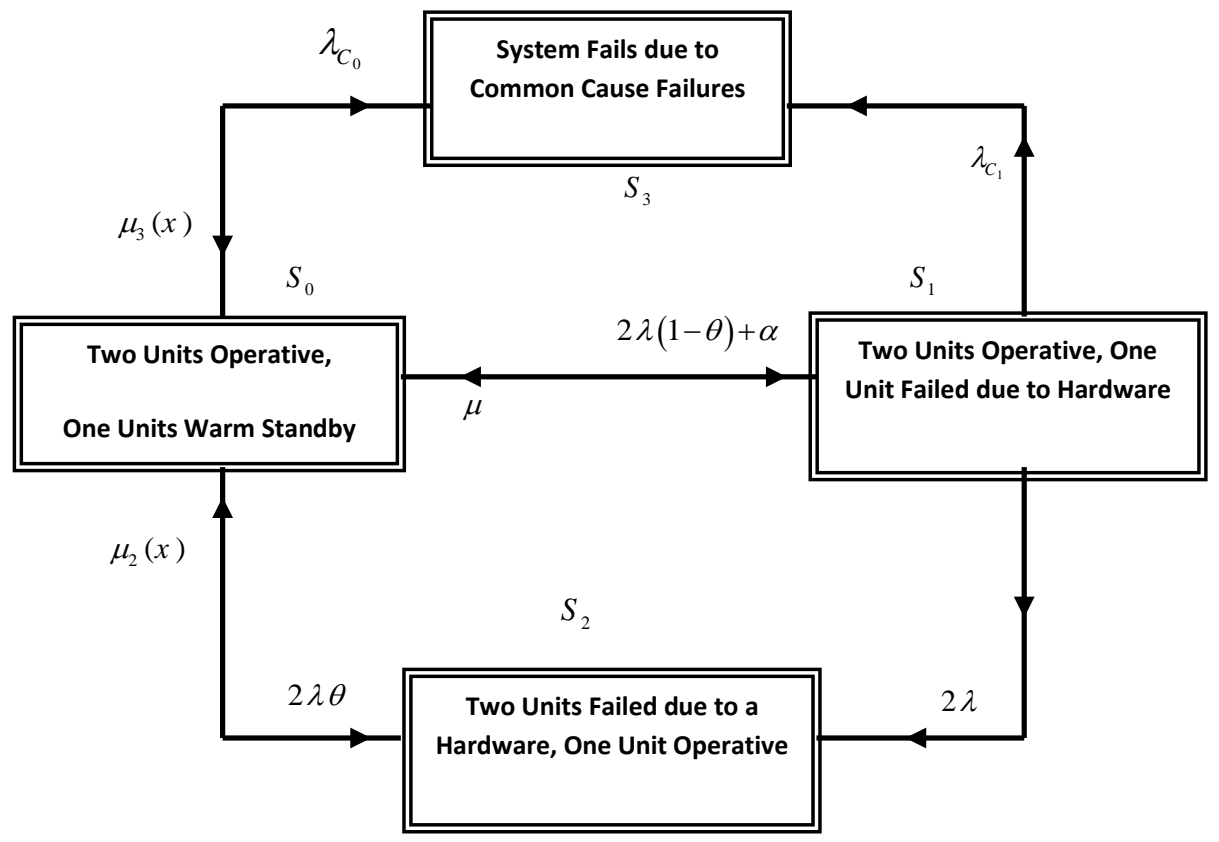

Fig 2: State-space diagram for configuration 2. 


\section{REFERENCES}

[1] Dhillon, B. S., Anude, O. C. 1993. Common-cause failure analysis of a non-identical unit parallel system with arbitrarily distributed repair times. Microelectronics and Reliability, Vol. 33, No. 1, pp. 87-103

[2] El-Said, K.M., El-Sherbeny, M.S. 2006. Comparing of reliability characteristic between two different systems. Applied Mathematics and Computation, Vol. 173, pp. 1183-99.

[3] El-Sherbeny, M.S. 2010. The optimal system for series systems with mixed standby components. Journal of Quality in Maintenance Engineering. Vol. 16 No. 3, 319334

[4] Galikowsky, C., Sivazlian, B. D., Chaovalitwongse, P. 1996. Optimal redundancies for reliability and availability of series systems. Microelectronics and Reliability, 36, 1537-1546
[5] Sridharan, V., \& Kalyani, T. V. 2002. Stochastic analysis of a non-identical two-unit parallel system with common-cause failure using GERT technique. Information and Management Sciences, 13, 49-57

[6] Shen, Z., Xiaoxiao, Hu., Weifeng, Fan. 2008. Exponential asymptotic property of a parallel repairable system with warm standby under common-cause failure. J. Math. Anal. Appl., 341, 457-466

[7] Wang, K. H., Kuo, C. C. 2000. Cost and probabilistic analysis of series systems with mixed standby components. Applied Mathematical Modelling, 24, 957967

[8] Wang, K. H., Pearn, W. L. 2003. Cost benefit analysis of series systems with warm standby components. Mathematical Methods of Operations Research, 58, 247258 